\title{
Abnormal resting-state brain activities in patients with first-episode obsessive-compulsive disorder
}

\author{
This article was published in the following Dove Press journal: \\ Neuropsychiatric Disease and Treatment \\ 16 February 2017 \\ Number of times this article has been viewed
}

\author{
Qihui Niu',* \\ Lei Yangl,* \\ Xueqin Song ${ }^{\prime}$ \\ Congying $\mathrm{Chu}^{2}$ \\ Hao Liu' \\ Lifang Zhang' \\ Yan $\mathrm{Li}^{\prime}$ \\ Xiang Zhang' \\ Jingliang Cheng' \\ Youhui Li' \\ 'Department of Psychiatry, The \\ First Affiliated Hospital, Zhengzhou \\ University, Zhengzhou, ${ }^{2}$ Brainnetome \\ Certer, Institute of Automation, \\ Chinese Academy of Sciences, Beijing, \\ People's Republic of China \\ *These authors contributed equally \\ to this work
}

Objective: This paper attempts to explore the brain activity of patients with obsessivecompulsive disorder (OCD) and its correlation with the disease at resting duration in patients with first-episode OCD, providing a forceful imaging basis for clinic diagnosis and pathogenesis of OCD.

Methods: Twenty-six patients with first-episode OCD and 25 healthy controls (HC group; matched for age, sex, and education level) underwent functional magnetic resonance imaging (fMRI) scanning at resting state. Statistical parametric mapping 8, data processing assistant for resting-state $\mathrm{fMRI}$ analysis toolkit, and resting state fMRI data analysis toolkit packages were used to process the fMRI data on Matlab 2012a platform, and the difference of regional homogeneity (ReHo) values between the OCD group and HC group was detected with independent two-sample $t$-test. With age as a concomitant variable, the Pearson correlation analysis was adopted to study the correlation between the disease duration and ReHo value of whole brain. Results: Compared with HC group, the ReHo values in OCD group were decreased in brain regions, including left thalamus, right thalamus, right paracentral lobule, right postcentral gyrus, and the ReHo value was increased in the left angular gyrus region. There was a negative correlation between disease duration and ReHo value in the bilateral orbitofrontal cortex (OFC).

Conclusion: OCD is a multifactorial disease generally caused by abnormal activities of many brain regions at resting state. Worse brain activity of the OFC is related to the OCD duration, which provides a new insight to the pathogenesis of OCD.

Keywords: OCD, resting state, functional MRI, regional homogeneity

\section{Introduction}

Obsessive-compulsive disorder (OCD) is a severe and debilitating neuropsychiatric disorder. The incidence of OCD is approximately $1 \%$ in adult males, $1.5 \%$ in adult females, and $2.7 \%$ in adolescents. ${ }^{1,2}$ A large sample epidemiological survey in the USA demonstrated that $2.3 \%$ of respondents meet the standards of OCD according to the Diagnostic and Statistical Manual of Mental Disorders, fourth edition (DSM-IV). ${ }^{3}$ The disease duration of OCD shows a gradual and chronic pattern. During a mean follow-up of 5.7 years, $40 \%$ of adolescent patients were found that OCD symptoms persisted with them, ${ }^{1}$ which lead to the impairment of cognitive function.

It has been suggested that dysregulation of the cortico-striatal-thalamo-cortical (CSTC) circuit serves as a pivotal pathophysiologic feature of OCD. ${ }^{4}$ Welch et al and Ahmari et al emphasized that the abnormal CSTC circuitry can induce OCD-like behaviors in a mouse model., 5 The study found that functional connectivity between the regional of orbitofrontal cortex (OFC) and external brain regions could be disordered in OCD patients. ${ }^{4,7}$ However, recent research found that CSTC is not the only important pathological basis of OCD; some other regions outside the CSTC circuitry,
Correspondence: Youhui Li; Jingliang Cheng

Department of Psychiatry, The First Affiliated Hospital, Zhengzhou University, I East Jianshe Road, Zhengzhou 450052, People's Republic of China

Tel +86 I 38 38I 2 8836;

+8613603863860

Email qiuliyouhui@I26.com; cjr.chjl@vip.I63.com
Neuropsychiatric Disease and Treatment 2017:13 507-513

507 
including parietal cortex and insula, are also involved in the pathogenesis of OCD, ${ }^{8}$ which suggests that the networks of OCD should be revisited.

The dysfunction of brain activity at resting state can explain why mental disorder patients commonly suffer from abnormal cognitive and emotional impairment to a certain extent. Resting-state functional magnetic resonance imaging has been developed extensively for clarifying the pathology of neurological disorders. Using statistical parametric maps, it was reported that OCD patients without treatment show a greater distant connectivity in the OFC and subthalamic nucleus and a greater local connectivity in the OFC and the putamen. ${ }^{9} \mathrm{~L}$ et al found an abnormal functional connectivity between the OFC and ventral anterior cingulate cortex in OCD patients with OFC platting as the center. ${ }^{7}$ A region of interest approach is commonly used to study the abnormal brain activity, while this method is limited in identifying other significative brain regions beyond the prevailing framework.

Regional homogeneity ( $\mathrm{ReHo}$ ) analysis is an indicator for measuring local synchronization of spontaneous activity within neighboring voxels at resting state. Since Zang et al proposed that ReHo analytic method is based on calculating the Kendall's coefficient of concordance in 2004, ${ }^{10}$ this method has been widely applied in evaluating neuropsychosis such as schizophrenia, bipolar disorder, Parkinson's disease, etc. ${ }^{11-13}$ Decreased ReHo indicates that functional activities of local brain lack coordination and are in a state of disorder, while increased ReHo shows a higher cooperation degree at the corresponding brain regions. In short, both increased and decreased ReHo indicate the disequilibrium of brain activity in certain regions at baseline, which leads to dysregulated emotion and behavior.

Therefore, the present study aimed to investigate the abnormal intrinsic brain activity of OCD patients at the resting state based on the whole brain, rather than CSTC circuit alone, using ReHo method. The correlation between brain activity and the duration of OCD was further reviewed.

\section{Materials and methods Subjects}

Written informed consent was provided by all subjects, and this study was approved by the Ethics Committee of the First Affiliated Hospital of Zhengzhou University. Twenty-six patients (18 males and 8 females) were diagnosed with first-episode OCD according to the criteria of DSM-IV in the First Affiliated Hospital of Zhengzhou University and recruited as the case group, and they had not received prior treatment with anti-OCD drugs or other psychotropic drugs from Aug 2013 to Dec 2014. Healthy volunteers (12 males and 13 females) were recruited as control group. The duration of OCD was confirmed by clinical interview. The inclusion criteria and exclusion criteria for patients with OCD and controls are shown in Table 1.

\section{Methods}

\section{Psychiatric scale assessment}

All subjects were required to complete the Yale-Brown Obsessive-Compulsive Scale (Y-BOCS), Hamilton Anxiety Rating Scale, and Hamilton Depression Rating Scale at psychology outpatient departments of the First Affiliated Hospital of Zhengzhou University. After the testing, all scales were collected on the spot and with calculated scores.

Table I Inclusion criteria and exclusion criteria for patients with OCD and controls

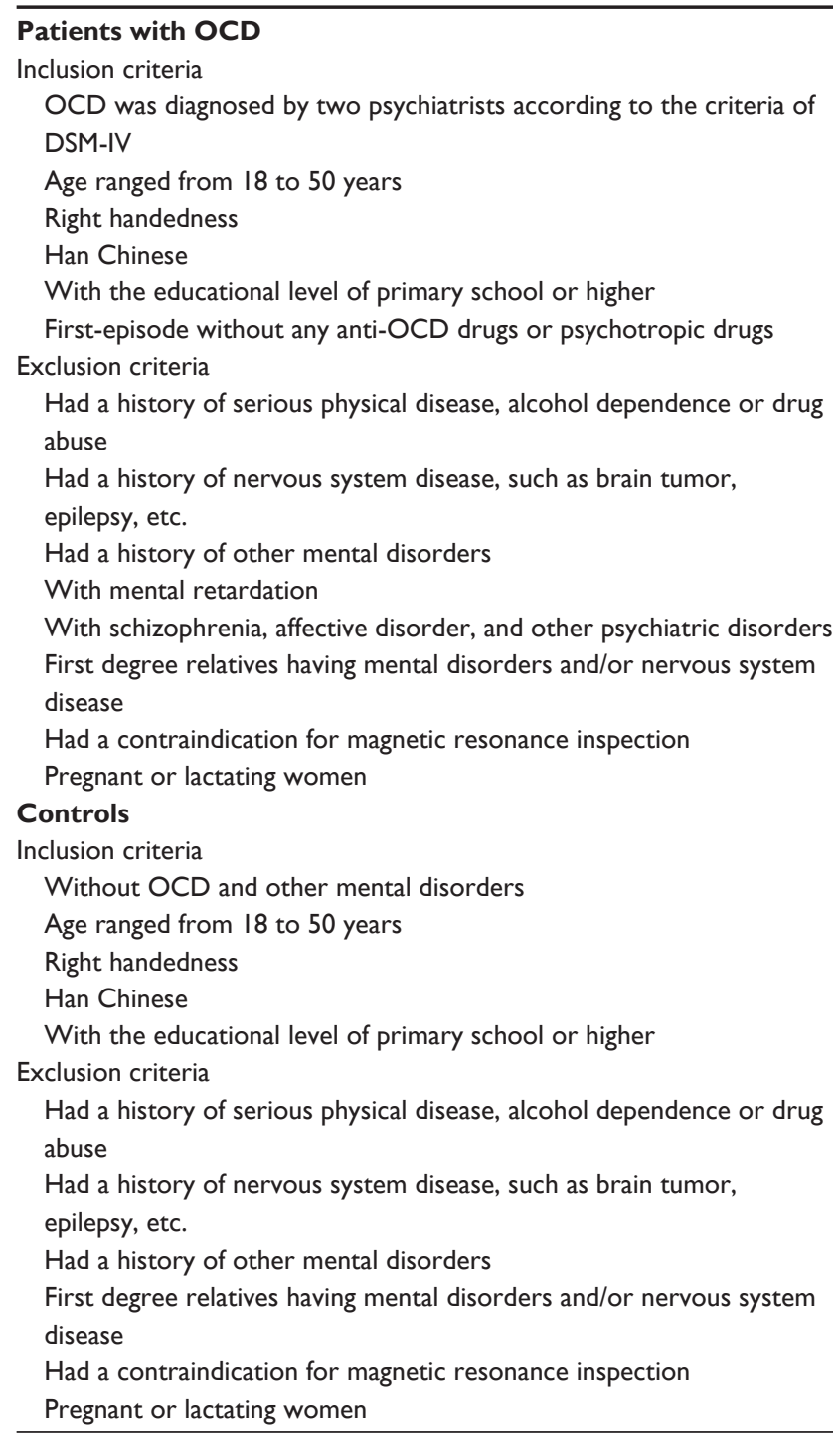

Abbreviations: DSM-IV, Diagnostic and Statistical Manual of Mental Disorders, fourth edition; OCD, obsessive-compulsive disorder. 
Magnetic resonance imaging (MRI) scanning

All subjects were scanned by 3.0 T MRI at MRI room in the First Affiliated Hospital of Zhengzhou University. Before scanning, subjects were told about the whole process of MRI scanning to ensure that they had a profound understanding. During the functional MRI (fMRI) scanning, participants were required to keep awake, breath peacefully, relax, and try to avoid any movement and thinking activities. A routine MRI scanning was performed firstly to exclude those subjects who had brain structural abnormalities and then data of fMRI at resting were collected.

\section{MRI date acquisition}

A GE MR750 3T MRI scanner with standard quadrature head coil was used to scan routinely and acquire fMRI-blood oxygenation level dependent (BOLD) data.

A routine MRI scanning including $\mathrm{T}_{1}$-weighted structural images and $\mathrm{T}_{2}$-weighted structural images, which were made firstly to exclude those subjects who had brain structural abnormalities and then data of fMRI at resting, were collected.

Resting state BOLD images of the whole brain were acquired by using gradient-echo echo-planar imaging sequence with the following parameters: repetition time $=2,000 \mathrm{~ms}$, echo time $=35 \mathrm{~ms}$, flip angle $=90^{\circ}$, matrix $=64 \times 64$, field of view $=240 \times 240 \mathrm{~mm}, 35$ slices (slices order: $1,3,5 \ldots 2,4,6 \ldots)$, thickness $/$ skip $=3.8 \mathrm{~mm} / 0 \mathrm{~mm}$, time point $=180$, and scan time $=6 \mathrm{~min}$.

\section{Image processing and analysis}

The preprocessing of fMRI-BOLD data was carried out using statistical parametric mapping 8, data processing assistant for resting-state fMRI analysis toolkit, and resting state fMRI data analysis toolkit based on Matlab 2012a platform. The first ten time points of each functional time series were deleted because of the possible inaccuracy caused by the unstable magnetic field or the participant's maladjustment. The remaining 170 time points of fMRI data were collected in subsequent preprocessing. The following steps were included: 1) Slice timing and head motion correction: correcting for within-scan acquisition time differences between slices and head motions. 2) Spatial normalization: normalizing the individual fMRI images to the standard template (each voxel was resampled to $3 \times 3 \times 3 \mathrm{~mm}^{3}$ ). 3) Removing the linear trend and band-pass filtering: a temporal filter $(0.01-0.08 \mathrm{~Hz})$ was used to reduce the effects of physiological noise. 4) Removing covariates: regressing out nuisance signals including the six head motion profiles, global mean signal, white matter signal, and cerebrospinal fluid signal.
By using the data processing assistant for resting-state fMRI analysis toolkit, ReHo value was calculated by using the Kendall coefficient of concordance to measure the similarity of time courses in 27 neighboring voxels. The standardized ReHo maps were normalized and smoothed using a Gaussian filter of $4 \mathrm{~mm}$ full width at half of the maximum value. Finally, each ReHo map was obtained by dividing the global mean value in the whole-brain mask, and the global mean ReHo was used for the following statistical analysis.

\section{Statistical analysis}

The independent two-sample $t$-test was applied to test the difference in ReHo values between the OCD patients and healthy controls (HC). Confounding factors including age, sex, and years of education were regressed out. The resultant $t$-value map was then thresholded using $P<0.005$ for each voxel and a cluster size of at least 14 voxels (uncorrected), and final statistical results were corrected using the AlphaSim program with a threshold of $P<0.005$. Pearson correlation was used to test the relationships between the mean ReHo values of the clusters of the case group and the duration of illness of OCD patients. The level of significance was set at $P<0.005$ and go through Alphasim correction with $P<0.005$. The processing results were overlaid and displayed on Colin 27 average brain template.

\section{Results}

\section{Demographic and clinical data of the participants}

After data filtering, a total of 51 subjects were enrolled in the study, including $26 \mathrm{OCD}$ patients and 25 controls. There were no differences in age, sex, and education level between the case group and control group (Table 2).

Table 2 Demographic and clinical data of the OCD group and HC

\begin{tabular}{lllll}
\hline Variables & $\mathbf{O C D}(\mathbf{n}=\mathbf{2 6})$ & $\mathbf{H C}(\mathbf{n}=\mathbf{2 5})$ & $\boldsymbol{t} / \chi^{2}$ & $P$-value \\
\hline Duration (months) & $65.88 \pm 74.31$ & & & \\
Sex (male/female) & $18 / 8$ & $12 / 13$ & 2.372 & 0.12 \\
Age (years) & $24.19 \pm 6.77$ & $22.68 \pm 4.96$ & 0.908 & 0.37 \\
Education (years) & $13.50 \pm 2.83$ & $15.04 \pm 3.32$ & -1.784 & 0.08 \\
BMI & $22.74 \pm 1.26$ & $22.20 \pm 1.1 \mathrm{I}$ & 1.64 & $0.1 \mathrm{I}$ \\
Y-BOCS total score & $22.92 \pm 6.82$ & $12.92 \pm 2.7 \mid$ & 6.94 & $0.00 *$ \\
HAMA total score & $14.35 \pm 8.16$ & $12.88 \pm 4.40$ & 0.80 & 0.43 \\
HAMD total score & $15.58 \pm 4.76$ & $14.56 \pm 4.43$ & 0.79 & 0.43 \\
\hline
\end{tabular}

Notes: Data expressed as mean \pm standard deviation. *Represents significant difference between $\mathrm{OCD}$ and $\mathrm{HC}$ group.

Abbreviations: BMI, body mass index; HAMA, Hamilton Anxiety Rating Scale; HAMD, Hamilton Depression Rating Scale; HC, healthy controls; OCD, obsessivecompulsive disorder; Y-BOCS, Yale-Brown Obsessive-Compulsive Scale. 
Table 3 Abnormal ReHo in OCD

\begin{tabular}{|c|c|c|c|c|c|c|}
\hline \multirow[t]{2}{*}{ Brain regions } & \multirow[t]{2}{*}{ BA } & \multicolumn{3}{|c|}{$\begin{array}{l}\text { MNI } \\
\text { coordinates }\end{array}$} & \multirow[t]{2}{*}{ Cluster } & \multirow[t]{2}{*}{ Peak T } \\
\hline & & $\mathbf{x}$ & $y$ & $\mathbf{z}$ & & \\
\hline \multicolumn{7}{|l|}{ OCD < HC } \\
\hline Thalamus- $R$ & I & 15 & -12 & 3 & 20 & 4.5832 \\
\hline Thalamus-L & I & -6 & -6 & 15 & 56 & 4.7513 \\
\hline Paracentral lobule- $R$ & 4 & 6 & -21 & 66 & 54 & 4.6517 \\
\hline Postcentral-R & 3 & 33 & -36 & 66 & 28 & 3.5865 \\
\hline \multicolumn{7}{|l|}{$O C D>H C$} \\
\hline Angular-L & 39 & -42 & -69 & 48 & 16 & -4.2166 \\
\hline
\end{tabular}

Notes: $P<0.005$, cluster size $>378 \mathrm{~mm}^{3}$, and Alphasim correction $(P<0.005)$. MNI coordinates: a three dimensional human brain coordinate positioning system made by MNI. "l' indicates no data.

Abbreviations: BA, Brodman area; HC, healthy controls; MNI, Montreal Neurological Institute; L, left side; $\mathrm{OCD}$, obsessive-compulsive disorder; $\mathrm{R}$, right side; ReHo, regional homogeneity; $x$, left-right direction; $y$, before-after direction, $z$, up-down direction.

\section{ReHo differences between OCD patients and controls}

Compared with healthy controls, decreased ReHo values were found in regions of right thalamus, left thalamus, right paracentral lobule- $\mathrm{R}$ and postcentral-R, and increased ReHo value was found in region of angular-L in OCD patients (Table 3; Figures 1 and 2).

\section{Correlation analysis between OCD duration and the ReHo of the whole brain}

A significant correlation between OCD duration and the ReHo value of the whole brain was observed in brain regions (Table 4; Figure 3).

\section{Discussion}

Based on the decreased ReHo value of bilateral thalamus, this study verified that the CSTC served as a nerve pathology foundation of OCD, which has been exactly shown in previous research. ${ }^{14,15}$ In CSTC, cortex is the key area to regulate emotion and behavior, striatum is the area for receiving cortical fiber and integrating signs from cortex, and thalamus plays a role in gating function of managing input and output information. If the gating function at the level of thalamus is inefficient, it will result in hyperactivity within the OFC (associated with intrusive thoughts) and hyperactivity within

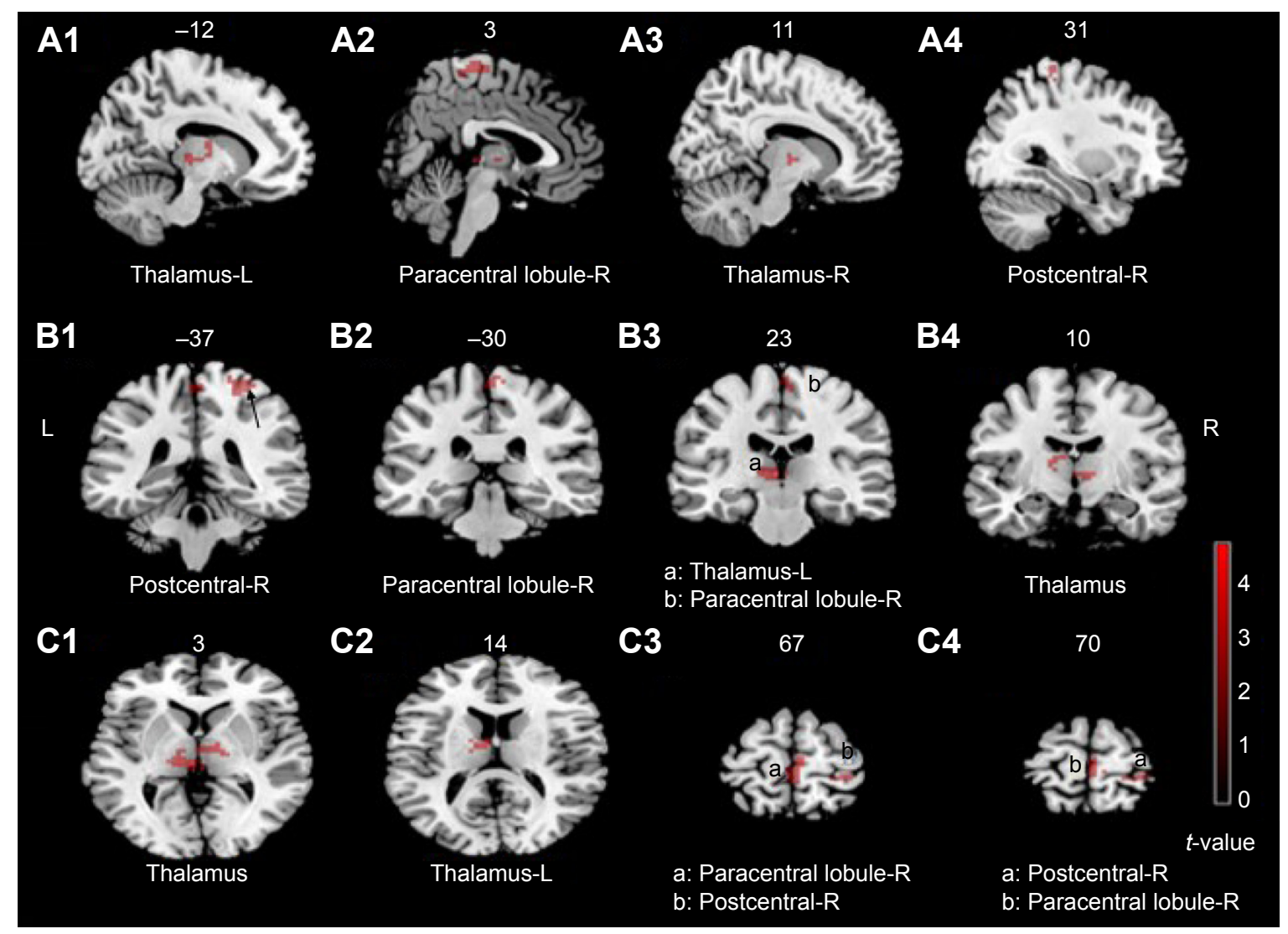

Figure I The brain regions showing decreased ReHo in OCD group.

Notes: These maps are the results of an independent two-sample $t$-test with $P<0.005$, cluster size $>378 \mathrm{~mm}^{3}$, and Alphasim correction $(P<0.005)$. The color bar indicates the $t$-values. Red denotes decreased ReHo including thalamus-R, thalamus-L, paracentral lobule-R, and postcentral-R. Al-A4 represent sagittal plane, BI-B4 represent coronal plane, and CI-C4 represent axial plane.

Abbreviations: OCD, obsessive-compulsive disorder; ReHo, regional homogeneity; R, right side; L, left side. 


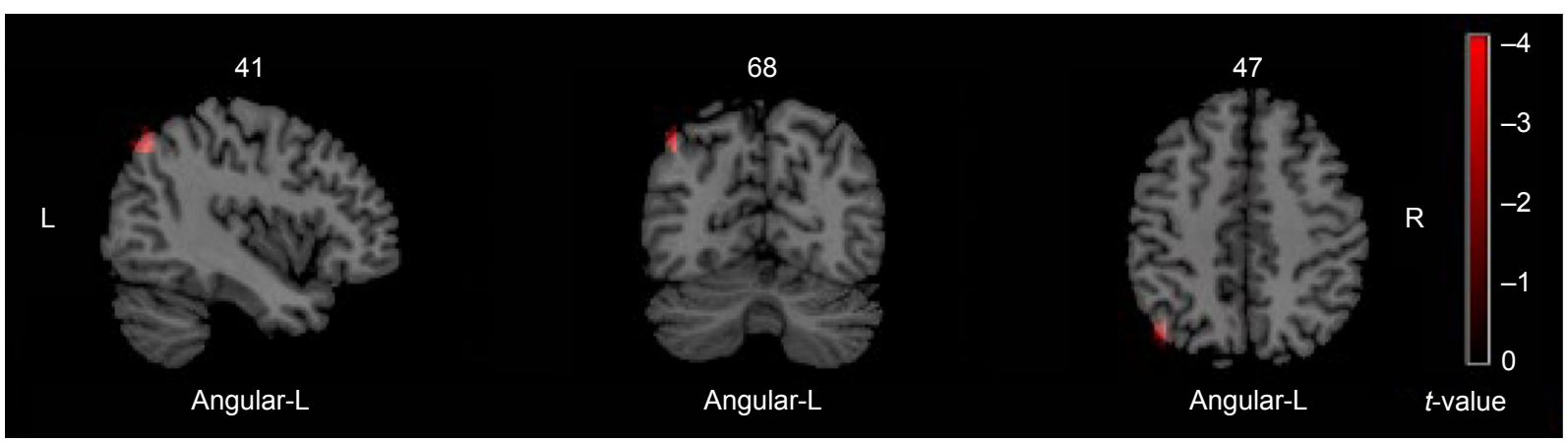

Figure 2 The brain regions showing increased ReHo in OCD group.

Notes: These maps are the results of an independent two-sample $t$-test with $P<0.005$, cluster size $>378 \mathrm{~mm}^{3}$, and Alphasim correction $(P<0.005)$. The color bar indicates the $t$-values. Red denotes increased ReHo including angular-L.

Abbreviations: OCD, obsessive-compulsive disorder; ReHo, regional homogeneity; R, right side; L, left side.

the anterior cingulate cortex (corresponding to nonspecific anxiety). Compulsion is viewed as a ritualistic behavior which can recruit the inefficient striatum in order to recover CSTC circuit, and reduce unwanted thoughts and alleviate anxiety. ${ }^{16}$ The dysfunction of CSTC is concerned with the emotion, behavior, and cognitive activity of OCD patients. ${ }^{4}$ It is noteworthy that thalamus acts as a key node in the circuits. Del Casale et al summarized the functional imaging studies of OCD and found that $20.4 \%$ of literature reported the alteration in thalamus of OCD patients. ${ }^{16}$ MRI perfusion-weighted imaging revealed that the relative cerebral blood flow of thalamus is increased in OCD group. ${ }^{17}$ An experimental study in the monkey suggested that overactivation of thalamus may provoke compulsive-like behaviors and neurovegetative manifestations, which are commonly associated with the feeling of anxiety in OCD patients. ${ }^{18}$

The discovery of abnormal brain activity in paracentral lobule-R, postcentral-R, and angular-L presents a new viewpoint that dysfunctional parietal is also involved in the nosogenesis of OCD, except for CSTC. ${ }^{19}$ Yang et al demonstrated that the patients with OCD showed higher ReHo value in the left anterior cingulate cortex, but lower ReHo value in the left inferior temporal gyrus, which supported the abnormal resting-state brain activity in drug-naïve OCD

Table 4 Correlation between OCD duration and the ReHo of the whole brain

\begin{tabular}{|c|c|c|c|c|c|}
\hline \multirow[t]{2}{*}{ Brain regions } & \multirow[t]{2}{*}{ BA } & \multicolumn{3}{|c|}{ MNI coordinates } & \multirow[t]{2}{*}{$\mathbf{r}$} \\
\hline & & $\mathbf{x}$ & $y$ & $\mathbf{z}$ & \\
\hline OFC & 12 & 2 & 16 & -15 & -0.71377 \\
\hline
\end{tabular}

Notes: $P<0.005$, cluster size $>297 \mathrm{~mm}^{3}$, and Alphasim correction $(P<0.005)$. MNI coordinates: a three dimensional human brain coordinate positioning system made by $\mathrm{MNI}$.

Abbreviations: BA, Brodman area; MNI, Montreal Neurological Institute; OFC, orbitofrontal cortex; OCD, obsessive-compulsive disorder; ReHo, regional homogeneity; $x$, left-right direction; $y$, before-after direction; $z$, up-down direction. patients. ${ }^{20}$ In the present study, decreased ReHo values were found in paracentral lobule- $\mathrm{R}$ and postcentral-R regions, and increased ReHo value was found in angular-L region. Postcentral and angular gyrus are located in the parietal lobe. Paracentral lobule is divided into anterior and posterior to the central sulcus. Dysfunctional paracentral lobule in this study was primarily located in the back of it, which participates mainly in the function of parietal lobe. Hence, it is considered that the decompensated function also exists in parietal lobe of OCD patients. The function of parietal lobe has been reported to be associated with skill learning, attention, and working memory, primarily. ${ }^{21}$ The reduced white matter volumes, abnormal cortical thickness, and modifications in glucose metabolism were found in parietal gyrus, ${ }^{8,22,23}$ and the metabolism of the parietal lobe was correlated with cognitive deficits in OCD patients. ${ }^{8}$ When OCD patients participated in a biological motion task, a reduced activation is shown in the postcentral gyrus. ${ }^{24}$ Parieto-temporal dysfunction was observed when a task with higher attentional load was devoted. ${ }^{25}$ The dysfunctional parietal activity might explain abnormal cognition of OCD patients to some extent.

In addition, abnormal brain activity in OFC may be a vulnerability marker for OCD. The correlation analysis showed that the ReHo value of orbitofrontal cortex was negatively related to the duration of OCD. Previous study also found an abnormal functional connectivity between the OFC and ventral anterior cingulate cortex in patients with OCD compared to HC. ${ }^{26}$ It has been demonstrated that the central 5-hydroxytryptamine might have more function decreased in patients with OCD, selective serotonin reuptake inhibitors showed positive efficacy in the treatment of OCD, and intact transmissions of 5-hydroxytryptamine have been connected with normal OFC functioning. ${ }^{27}$ Although the related researches about $\mathrm{OFC}$ are numerous, the results 


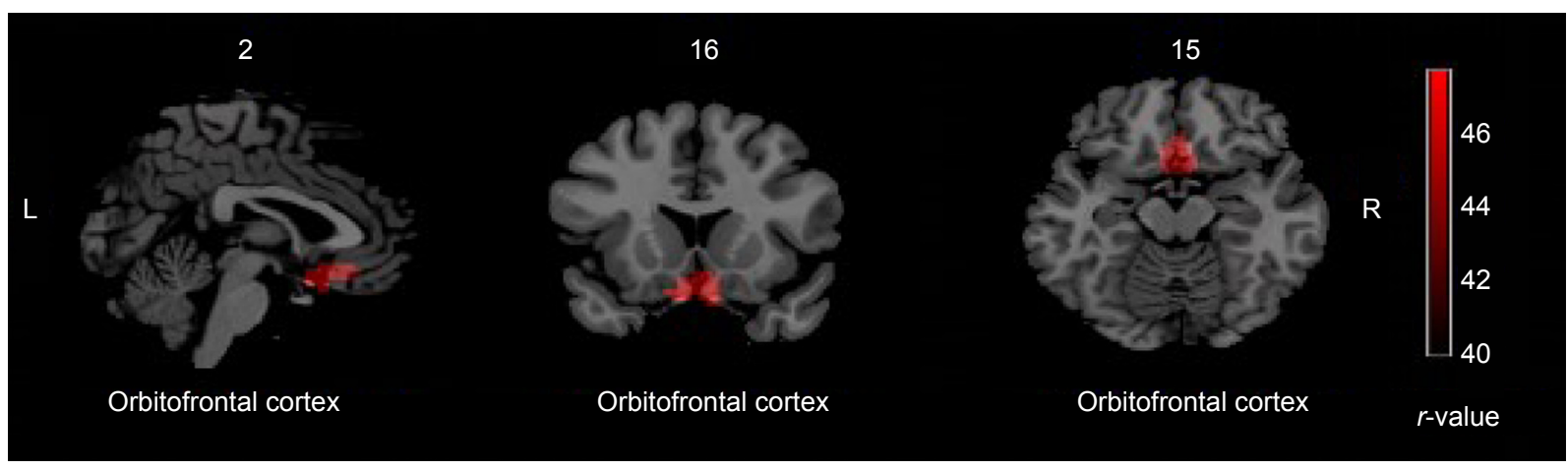

Figure 3 Correlation between OCD duration and the ReHo of the whole brain.

Notes: These maps are the results of correlation analysis between OCD duration and the ReHo with $P<0.005$, cluster size $>297 \mathrm{~mm}^{3}$, and Alphasim correction $(P<0.005)$. The color bar indicates the $r$-values. Red denotes that the region of ReHo was negatively correlated with OCD duration including OFC.

Abbreviations: OFC, orbitofrontal cortex; OCD, obsessive-compulsive disorder; ReHo, regional homogeneity; R, right side; L, left side.

show inconsistency. A genetic association study showed that patients with OCD and their first-degree relatives exhibited overlapping increased functional connectivity strength in left OFC. ${ }^{4}$ The strongest trend toward association was identified between two single nucleotide polymorphisms in DLGAP2 (glutamatergic candidate genes) and OFC white matter volume ${ }^{28}$ The altered structural connectivity of fibers between the OFC and the striatum partly leads to the pathophysiology of OCD. ${ }^{29}$ However, the results of Rotge's et al study showed that OFC dysfunction might not be the contributor to OCD symptoms. ${ }^{30}$ Ahmari et al also demonstrated that acute OFCventromedial striatum stimulation did not produce repetitive behaviors, but generates a progressive increase in grooming, a mouse behavior related to OCD. ${ }^{6}$ In this study, abnormal activity was not found in OFC based on the whole brain ReHo analysis but a negative correlation between the ReHo value of OFC and the duration of illness was identified. These findings suggested that the dysfunction of OFC might be a quantitative alternation, which showed that a longer disease course had greater alteration in the functions of OFC.

\section{Conclusion}

OCD is a multifactorial disease which involves many brain regions having abnormal activities at resting state. This study not only supports that the disorganized CSTC plays a role in the pathogenesis of OCD but also proposes that parietal lobe is also closely associated with the occurrence of OCD. As the disease progresses, the dysfunction of OFC tends to be more obvious. The multiple dysfunction brain activity might contribute to the pathophysiological understanding of OCD and bring new insights to the pathogenesis of OCD syndromes. However, this study is limited due to the small sample size, without the analysis combined with structural magnetic resonance imaging, and lack of observation in patients after the treatment.

\section{Acknowledgments}

Funding for this study was provided by the Science and Technology Agency of Henan (No 142102310512) and the West China Psychiatric Association (Wcpafund-201608).

\section{Disclosure}

The authors report no conflicts of interest in this work.

\section{References}

1. Maduro JH, Pras E, Willemse PH, de Vries EG. Acute and long-term toxicity following radiotherapy alone or in combination with chemotherapy for locally advanced cervical cancer. Cancer Treat Rev. 2003; 29(6):471-488.

2. Soomro GM. Obsessive compulsive disorder; 2012. Available from: https://www.ncbi.nlm.nih.gov/pubmed/22305974. Accessed August 24, 2016.

3. Ruscio AM, Stein DJ, Chiu WT, Kessler RC. The Epidemiology of obsessive-compulsive disorder in the national comorbidity survey replication. Mol Psychiatry. 2010;15(1):53-63.

4. Hou JM, Zhao M, Zhang W, Song LH, Wu WJ, Wang J, et al. Resting-state functional connectivity abnormalities in patients with obsessive-compulsive disorder and their healthy first-degree relatives. J Psychiatry Neurosci. 2014;39:304-311.

5. Welch JM, Lu J, Rodriguiz RM, et al. Cortico-striatal synaptic defects and OCD-like behaviours in Sapap3-mutant mice. Nature. 2007;448: 894-900.

6. Ahmari SE, Spellman T, Douglass NL, et al. Repeated corticostriatal stimulation generates persistent OCD-like behavior. Science. 2013;340(6137):1234-1239.

7. Ping L, Sufang L, Haiying H, et al. Abnormal spontaneous neural activity in obsessive-compulsive disorder: a resting-state functional magnetic resonance imaging study. PLoS One. 2013;8(6):1591-1594.

8. Millet B, Dondaine T, Reymann JM, et al. Obsessive compulsive disorder networks: positron emission tomography and neuropsychology provide new insights. PLoS One. 2013;8(1):e53241.

9. Beucke JC, Sepulcre J, Talukdar T, et al. Abnormally high degree connectivity of the orbitofrontal cortex in obsessive-compulsive disorder. JAMA Psychiatry. 2013;70(6):619-629.

10. Zang Y, Jiang T, Lu Y, He Y, Tian L. Regional homogeneity approach to fMRI data analysis. Neuroimage. 2004;22(1):394-400.

11. Chyzhyk D, Graña M, Öngür D, Shinn AK. Discrimination of schizophrenia auditory hallucinators by machine learning of resting-state functional MRI. Int J Neural Syst. 2015;25(3):1550007. 
12. Gao W, Jiao Q, Lu S, et al. Alterations of regional homogeneity in pediatric bipolar depression: a resting-state fMRI study. BMC Psychiatry. 2014; 14:222.

13. Sheng K, Fang W, Su M, et al. Altered spontaneous brain activity in patients with Parkinson's disease accompanied by depressive symptoms, as revealed by regional homogeneity and functional connectivity in the prefrontal-limbic system. PLoS One. 2014;9(1):e84705.

14. Posner J, Marsh R, Maia TV, Peterson BS, Gruber A, Simpson HB. Reduced functional connectivity within the limbic cortico-striatothalamo-cortical loop in unmedicated adults with obsessive-compulsive disorder. Hum Brain Mapp. 2014;35(6):2852-2860.

15. Brennan BP, Rauch SL, Jensen JE, Pope HG Jr. A critical review of magnetic resonance spectroscopy studies of obsessive-compulsive disorder. Biol Psychiatry. 2013;73(1):24-31.

16. Del Casale A, Kotzalidis GD, Rapinesi C, et al. Functional neuroimaging in obsessive-compulsive disorder. Neuropsychobiology. 2011; 64(2):61-85.

17. Chen XL, Xie JX, Han HB, Cui YH, Zhang BQ. MR perfusion-weighted imaging and quantitative analysis of cerebral hemodynamics with symptom provocation in unmedicated patients with obsessive-compulsive disorder. Neurosci Lett. 2004;370(2-3):206-211.

18. Rotge JY, Aouizerate B, Amestoy V, et al. The associative and limbic thalamus in the pathophysiology of obsessive-compulsive disorder: an experimental study in the monkey. Transl Psychiatry. 2012;2:e161.

19. Jose D, Narayanaswamy JC, Agarwal SM, Kalmady SV, Venkatasubramanian G, Reddy YCJ. Corpus callosum abnormalities in medicationnaïve adult patients with obsessive compulsive disorder. Psychiatry Res Neuroimaging. 2015;231:341-345.

20. Yang $\mathrm{T}$, Cheng $\mathrm{Y}$, Li H, et al. Abnormal regional homogeneity of drug-naive obsessive-compulsive patients. Neuroreport. 2010;21(11): 786-790.
21. Cabeza R, Nyberg L. Neural bases of learning and memory: functional neuroimaging evidence. Current Opin Neurol. 2000;13(4):415-421.

22. Peng Z, Shi F, Shi C, et al. Structural and diffusion property alterations in unaffected siblings of patients with obsessive-compulsive disorder. PLoS One. 2014;9(1):e85663.

23. Piras F, Piras F, Chiapponi C, Girardi P, Caltagirone C, Spalletta G. Widespread structural brain changes in OCD: a systematic review of voxel-based morphometry studies. Cortex. 2015;62:89-108.

24. Jung WH, Gu BM, Kang DH, et al. BOLD response during visual perception of biological motion in obsessive-compulsive disorder. Eur Arch Psychiatry Clin Neurosci. 2009;259(1):46-54.

25. Page LA, Rubia K, Deeley Q, et al. A functional magnetic resonance imaging study of inhibitory control in obsessive-compulsive disorder. Psychiatry Res. 2009;174(3):202-209.

26. Ping L, Su-Fang L, Hai-Ying H, et al. Abnormal spontaneous neural activity in obsessive-compulsive disorder: a resting-state functional magnetic resonance imaging study. PLoS One. 2013;8(6):e67262.

27. El Mansari M, Blier P. Responsiveness of 5-HT(1A) and 5-HT2 receptors in the rat orbitofrontal cortex after long-term serotonin reuptake inhibition. J Psychiatry Neurosci. 2005;30(4):268-274.

28. Ke W, Hanna GL, Easter P, Kennedy JL, Rosenberg DR, Arnold PD. Glutamate system genes and brain volume alterations in pediatric obsessive-compulsive disorder: a preliminary study. Psychiatry Res. 2013;211(3):214-220.

29. Nakamae T, Sakai Y, Abe Y, et al. Altered fronto-striatal fiber topography and connectivity in obsessive-compulsive disorder. PLoS One. 2014;9:e112075

30. Rotge JY, Langbour N, Dilharreguy B, et al. Contextual and behavioral influences on uncertainty in obsessive-compulsive disorder. Cortex. 2015;62:1-10
Neuropsychiatric Disease and Treatment

\section{Publish your work in this journal}

Neuropsychiatric Disease and Treatment is an international, peerreviewed journal of clinical therapeutics and pharmacology focusing on concise rapid reporting of clinical or pre-clinical studies on a range of neuropsychiatric and neurological disorders. This journal is indexed on PubMed Central, the 'PsycINFO' database and CAS,

\section{Dovepress}

and is the official journal of The International Neuropsychiatric Association (INA). The manuscript management system is completely online and includes a very quick and fair peer-review system, which is all easy to use. Visit http://www.dovepress.com/testimonials.php to read real quotes from published authors. 